\title{
RPS12-specific shRNA inhibits the proliferation, migration of BGC823 gastric cancer cells with S100A4 as a downstream effector
}

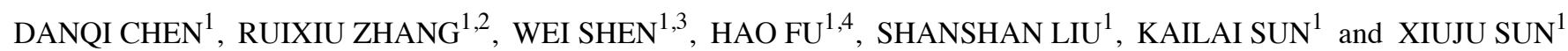 \\ ${ }^{1}$ Department of Medical Genetics, China Medical University, Shenyang 110001; ${ }^{2}$ Department of Molecular Oncology, \\ Eastern Hepatobiliary Surgery Hospital, The Second Military Medical University of Chinese PLA, Shanghai 200438; \\ Departments of ${ }^{3}$ Pathophysiology and ${ }^{4}$ Biochemistry, Shenyang Medical College, Shenyang 110034, P.R. China
}

Received January 4, 2013; Accepted March 6, 2013

DOI: $10.3892 /$ ijo.2013.1872

\begin{abstract}
Our previous study using suppression subtractive hybridization (SSH), cDNA microarray and semi-quantitative RT-PCR showed that RPS12 was overexpressed in gastric cancer and it was closely related to metastasis. However, the role of RPS12 in gastric cancer is not clear, which led us to conduct the current study to further investigate the effects of RPS12 on the proliferation and migration of gastric cancer cells, and also to explore the underlying molecular mechanisms. RNA interference was used to inhibit the expression of RPS12. The expression of RPS12 and S100A4 in gastric cancer cells was determined using semi-quantitative RT-PCR and western blot analysis. Cell proliferation and migration were detected by MTT and transwell assay, respectively. In addition, the promoter activity of S100A4 was measured by a Dual-Luciferase Reporter Assay System. We found that RNAi-mediated RPS12 downregulation led to reduced proliferation and migration of BGC823 and SGC7901 gastric cancer cells. Further results showed that RPS12 inhibition led to reduced $S 100 A 4$ expression and decreased promoter activity of $5100 A 4$ in BGC823 cells. We demonstrated that ectopic expression of $S 100 A 4$ reversed the reduced proliferation and migration ability after RPS12 inhibition in BGC823 cells. Our findings provide the first demonstration that RPS12 plays important roles in regulating the proliferation and migration of gastric cancer cells. S100A4 can mediate the effects of RPS12 as a downstream effector.
\end{abstract}

\section{Introduction}

Despite its declining incidence, gastric cancer remains a worldwide health problem that is the second leading cause

Correspondence to: Professor Xiuju Sun, Department of Medical Genetics, China Medical University, No. 92 Bei Er Road, Heping District, Shenyang 110001, P.R. China

E-mail: xjsun@mail.cmu.edu.cn

Key words: RPS12, gastric cancer, RNA interference, migration, proliferation of cancer-related death with little improvement of long-term survival during the past decades. In order to improve patient prognosis and therapy, it is now mandatory to further understand the molecular features involved in the pathogenesis. Although it has been under intensive investigation, the molecular mechanism of gastric cancer is not well understood and needs further exploring.

In an attempt to identify new factors involved in gastric cancer, we previously performed a comprehensive analysis of differentially expressed genes in gastric cancer by SSH (1). RPS12 was identified as one of the genes overexpressed in gastric cancer compared with the corresponding non-tumorous tissue, which was further confirmed by RT-PCR and DOT blot analysis in more samples. Clinicopathological analysis revealed that the enhanced RPS12 expression was related to metastasis of gastric cancer (1). Subsequent cDNA microarray studies showed that RPS12 expression in lymph node metastases was much higher than that in the primary gastric cancers (2). All the above results suggested that RPS12 might play important roles in the progression and metastasis of gastric cancer, which led us to investigate the function of RPS12 in gastric cancer cells. Our previous study showed that suppression of RPS12 expression by RNAi led to increased apoptosis of gastric cancer cells (3). In the current study, we focused on further investigating the effects of RPS12 on the proliferation and migration of gastric cancer cells, and the underlying mechanisms by exploring the downstream effector of RPS12.

\section{Materials and methods}

Cell culture. The human gastric cancer cell line BGC823 and SGC7901 were cultured in RPMI-1640 medium (Invitrogen, Carlsbad, CA, USA) supplemented with $10 \%$ fetal bovine serum in a humidified $37^{\circ} \mathrm{C}$ incubator with $5 \% \mathrm{CO}_{2}$.

Construction and transfection of RPS12-specific short hairpin RNA (shRNA) expression vector. Two different RPS12 hairpin oligonucleotides (oligos) were designed by selecting appropriate sequences from the human RPS12 mRNA according to the online software of Ambion Inc. (Austin, TX, USA). The 
first one named RPS12-shRNA-A oligo (sense: 5'-GATCCagtg gttggttgcagttgt $T T C A A G A G A$ acaactgcaaccaaccacttt $\mathrm{A}-3^{\prime}$; antisense: 5'-AGCTTaaagtggttggttgcagttgt $T C T C T T G A A$ acaactgcaaccaaccact $\mathrm{G}^{-} 3^{\prime}$ ) contained a region specific to bases 386-406 of RPS12 mRNA (marked in bold); the second one named RPS12-shRNA-B oligo (sense: 5'-GATCCgctgcca aagcettagaca $T T C A A G A G A \operatorname{tgtctaaggctttggcagctt} \mathrm{A}-3^{\prime}$; antisense: 5'-AGCTTaagctgccaaagccttagacaTCTCTT GAAtgtctaaggetttggcagc $\mathrm{G}-3^{\prime}$ ) contained a region specific to bases 192-212 of RPS12 mRNA (marked in bold). The RPS12-shRNA-A or RPS12-shRNA-B oligo contained a hairpin loop region (italicized), and $5^{\prime}$ and $3^{\prime}$ linker sequences for subcloning into the BamHI and HindIII sites of the pSliencer 4.1-CMV neo vectors. Each oligo was annealed with its complementary strand, and the resulting double-stranded shRNA oligo was cloned into the pSilencer 4.1-CMV neo vector (Ambion Inc.) making $\mathrm{pRPS12-shRNA-A}$ or $\mathrm{pRPS12-shRNA-B}$ according to the manufacturer's instructions. The control shRNA expression vector (pControl-shRNA) was the same as previously reported (4). Competent JM109 bacteria (Takara, Japan) were transformed with $\mathrm{pRPS12-shRNA-A/B}$ or pControl-shRNA. The plasmids were prepared from individual bacterial colonies, and confirmed by sequencing analysis.

pRPS12-shRNA-A/B or pControl-shRNA was transfected into BGC823 cells respectively, using Lipofectamine ${ }^{\mathrm{TM}} 2000$. We selected the more efficient one in inhibiting RPS12 mRNA

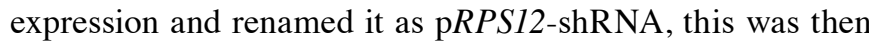
used to inhibit RPS12 expression in BGC823 and SGC7901 cells in the subsequent studies.

The cells transfected with $\mathrm{pRPS12-shRNA}$ or pControlshRNA were referred to as BGC823/SGC7901/pRPS12-shRNA cells or BGC823/SGC7901/pControl-shRNA cells, respectively.

RNA extraction and semi-quantitative RT-PCR. Total RNA of the cells was extracted using TRIzol reagent (Invitrogen). The reverse transcription reaction was performed using the FirstStrand cDNA synthesize kit (Promega, Madison, WI, USA) with $1 \mu \mathrm{g}$ of RNA in a final volume of $20 \mu \mathrm{l}$. The newly synthesized cDNA was amplified by PCR. Primers specific for human RPS12, S100A4 or $\beta$-actin were designed as follows: RPS12 sense 5'-ggCTTgggTgCgTTCAAgAT-3', antisense 5'-ggCCT gAgACTCCTTgCCA-3'; S100A4 sense 5'-gATgT gATggTgTC CACCTT-3', antisense 5'-ATTTCTTCCTgggCTgCTTA-3'; $\beta$-actin sense 5'-CTCTTCCagCCTTCCTTCCT-3', antisense 5'-CACCTTCACCgTTCCAgTTT-3'. Amplication cycles were: $95^{\circ} \mathrm{C}$ for $5 \mathrm{~min}$, then 30 cycles at $95^{\circ} \mathrm{C}$ for $30 \mathrm{sec}, 58^{\circ} \mathrm{C}$ for $30 \mathrm{sec}$ and $72^{\circ} \mathrm{C}$ for $30 \mathrm{sec}$, followed by $72^{\circ} \mathrm{C}$ for $10 \mathrm{~min}$. Aliquots of PCR product were checked by electrophoresis on $1.5 \%$ agarose gels, and the fragments were visualized by ethidium bromide staining. The experiments were performed three times.

Western blot analysis. Cells were washed with ice-cold PBS after being trypsinized, and then centrifuged at 1,000 rpm for $5 \mathrm{~min}$ at $4^{\circ} \mathrm{C}$. The pellet containing $10^{6}$ cells was lysed in $100 \mu \mathrm{l}$ of lysis buffer $(50 \mathrm{mM}$ Tris, $\mathrm{pH} 7.2,1 \%$ Triton $\mathrm{X}-100,0.5 \%$ sodium deoxycholate, $0.1 \%$ SDS, $500 \mathrm{mM} \mathrm{NaCl}, 10 \mathrm{mM} \mathrm{MgCl}_{2}$ with $10 \mu \mathrm{g} / \mathrm{ml}$ leupeptin, $10 \mu \mathrm{g} / \mathrm{ml}$ aprotinin and $1 \mathrm{mM}$ PMSF) and quantified by Bradford method. Total protein $(100 \mu \mathrm{g})$ was separated by SDS-PAGE (12\% polyacrylamide gel) and transferred to polyvinylidene difluoride (PVDF) membranes (Bio-Rad).
The membranes were incubated in blocking solution consisting of 5\% non-fat milk in TBST (10 mM Tris- $\mathrm{HCl}(\mathrm{pH} 8.0)$, $150 \mathrm{mM} \mathrm{NaCl}$, and $0.1 \%$ Tween-20) at room temperature for $3 \mathrm{~h}$, then immunoblotted with primary antibodies such as anti-RPS12 (1:800 dilution; Proteintech), anti-S100A4 (1:1,000 dilution; Lab Vision) and anti- $\beta$-actin (1:1,500 dilution; Santa Cruz) respectively, followed by incubation with a peroxidaseconjugated second antibody (goat anti-rabbit IgG for RPS12, S100A4 and $\beta$-actin). The reagent for enhanced chemiluminescence (Amersham, Freiburg, Germany) was used for detection and developed on X-ray film. The experiments were performed three times.

MTT assay. BGC823/SGC7901/pRPS12-shRNA cells and BGC823/SGC7901/pControl-shRNA cells at 12 h to 3 days after transfection were plated in 96-well plates at a density of $5 \times 10^{3}$ cells per well and incubated at $37^{\circ} \mathrm{C}$ in $5 \% \mathrm{CO}_{2}$. At different time points (day 3, 4, 5, 6, 7 and 8 after transfection), the proliferation ability was assayed by using 3-(4,5-dimethylthiazol2-yl)-2,5-diphenyltetrazolium bromide (MTT) as previously reported (4). The absorbance was read at a wavelength of $570 \mathrm{~nm}$ in Multiscan MC microplate reader. Each experiment was performed in triplicate and repeated three times.

Transwell assay. For cell migration assays, 5x10 4 BGC823/ SGC7901/pRPS12-shRNA cells and BGC823/SGC7901/ pControl-shRNA cells at 4th day after transfection were trypsinized, washed, resuspended in serum-free RPMI-1640, and plated in the upper chamber of $8-\mu \mathrm{m}$ pore transwells (Costar, USA), respectively. The lower chamber was filled with RPMI-1640 supplemented with $10 \%$ FBS as a chemoattractant. Cells were incubated at $37^{\circ} \mathrm{C}$ for $24 \mathrm{~h}$. Non-migrated cells on the upper surface of the insert membrane were removed with a cotton swab. Migrated cells on the lower surface were washed with PBS, fixed with $10 \%$ formaldehyde, stained, photographed and counted under high-power magnification. Five random fields were analyzed for each chamber. The experiments were performed three times.

Luciferase reporter assay. The plasmid pGL3-S100A4-luc vector containing a regulatory fragment located in the promoter region and the first intron of $5100 A 4$ gene (from -387 to +550 ), was constructed in a previous study by our group (5). On day 4 after transfection of RPS12-specific shRNA, BGC823/RPS12shRNA cells at a density of $1.5 \times 10^{4}$ cells/well in a 24 -well plate were transfected with $0.8 \mu \mathrm{g}$ of the pGL3-S100A4-luc or pGL3-basic-luc constructs, with Renilla luciferase (Promega) as the internal control (5). After incubation for $24 \mathrm{~h}$, the cells were harvested, and luciferase activity was measured using a DualLuciferase Reporter Assay System (Promega) and normalized by the internal control values. The experiments were performed three times.

Rescue assay after ectopic expression of S100A4 in BGC823/ pRPS12-shRNA cells. To investigate the effect of S100A4 on gastric cancer cells after RPS12 suppression, at $48 \mathrm{~h}$ after RPS12-sepcific shRNA transfection, BGC823/RPS12-shRNA cells were transfected with $4 \mu \mathrm{g}$ pcDNA3.1-S100A4 (6) using Lipofectamine 2000, with pcDNA3.1-empty vector transfected cells as a control. At different time points, cells were harvested 

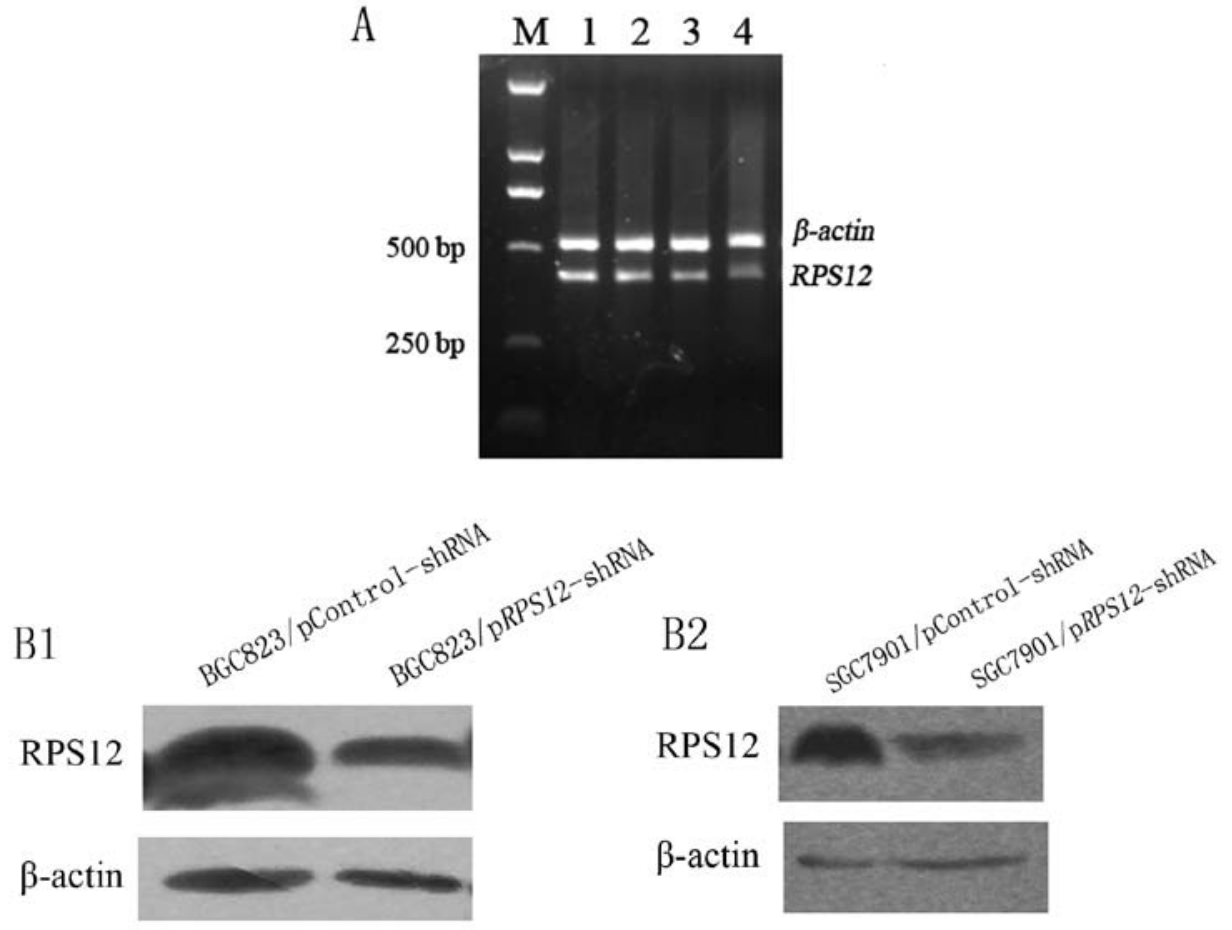

Figure 1. Downregulation of RPS12 expression in gastric cancer cells by RNAi. (A) Semi-quantitative RT-PCR analysis of RPS12 mRNA level in BGC823 cells on day 5 after transfection with pRPS12-shRNA-A or pRPS12-shRNA-B. Lane M, DNA marker-DL2000; lane 1, parental BGC823; lane 2, pControl-shRNA transfection; lanes 3 and 4,pRPS12-shRNA-A and pRPS12-shRNA-B transfection, respectively. (B) Expression of RPS12 protein in (B1) BGC823 and (B2) SGC7901 cells on day 5 after transfection of $\mathrm{pRPS12}$-shRNA was analyzed by western blot analysis as described in Materials and methods.

for determining the proliferation, and migration by MTT and transwell assay, respectively, as described above.

Statistical analysis. The data of absorbance in MTT, cell numbers in transwell and luciferase activities in luciferase reporter assay are presented as the means \pm SD. Statistical analyses were carried out using the Statistical Package for the Social Sciences (SPSS Inc., Chicago, IL, USA), where $\mathrm{p}<0.05$ was considered significant.

\section{Results}

Downregulation of RPS12 expression in gastric cancer cells by RNAi. We first evaluated the efficiency of the two shRNAs specific to different regions of RPS12 in silencing RPS12 expression on day 5 after transfection by using semi-quantitative RT-PCR (Fig. 1A). The results showed that RPS12 mRNA expression in $\mathrm{pRPS12}$-shRNA-B transfected BGC823 cells was obviously lower than that in $\mathrm{pRPS12-shRNA-A}$ transfected cells indicating that $\mathrm{p} R P S 12$-shRNA-B can suppress RPS12 expression more efficiently than pRPS12-shRNA-A in BGC823 cells. Therefore, we selected RPS12-shRNA-B (renamed RPS12-shRNA) to inhibit RPS12 expression in BGC823 and SGC7901 cells in the subsequent studies, and observed the cellular effects mainly at/or around day 5 after RPS12-shRNA transfection.

The results of western blot analysis showed that RPS12 protein expression in BGC823/SGC7901/pRPS12-shRNA cells at day 5 after transfection was obviously lower than that in BGC823/SGC7901/pControl-shRNA cells (Fig. 1B).
Downregulation of RPS12 expression leads to decreased proliferation and migration of gastric cancer cells. We observed the effects of RPS12 inhibition on the proliferation and migration of BGC823 and SGC7901 cells. The MTT assay showed that BGC823/SGC7901/pRPS12-shRNA cells displayed significantly reduced proliferation, as compared to BGC823/ SGC7901/pControl-shRNA cells at different time points after transfection of the corresponding shRNA (Fig. 2A). Transwell assays revealed that the number of migrated BGC823/SGC7901/ pRPS12-shRNA cells $(46.2 \pm 9.45,54.68 \pm 8.43)$ was significantly fewer than that of their corresponding pControl-shRNA cells $(118.05 \pm 15.78,78.4 \pm 27.49)(\mathrm{p}<0.05)($ Fig. 2B and C).

Effect of RPS12 inhibition on S100A4 expression in BGC823 cells. Having demonstrated that RPS12 plays important roles in regulating proliferation and migration of gastric cancer cells, we attempted to investigate the molecular mechanism through which RPS12 exerts its functions. It has been demonstrated by our group that $S 100 A 4$ plays crucial roles in regulating proliferation of gastric cancer cells (4), and also the migration of the cells (unpublished). We hypothesized that S100A4 might mediate the effect of RPS12 as its downstream target. To test our hypothesis, S100A4 expression was determined by RT-PCR and western blot analysis after RPS12 inhibition. We found that BGC823/pRPS12-shRNA cells displayed an obviously decreased S100A4 mRNA and protein expression compared with BGC823/pControl-shRNA cells (Fig. 3).

Effect of RPS12 inhibition on the transcriptional activity of S100A4 promoter in BGC823 cells. In order to investigate 
A1

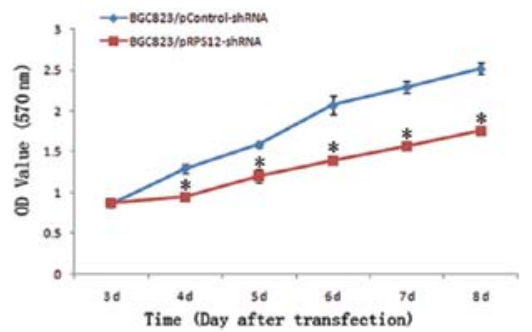

B1

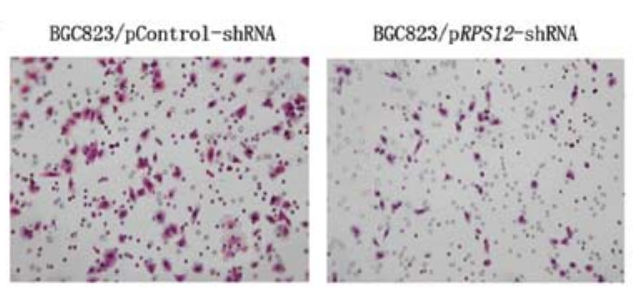

C1

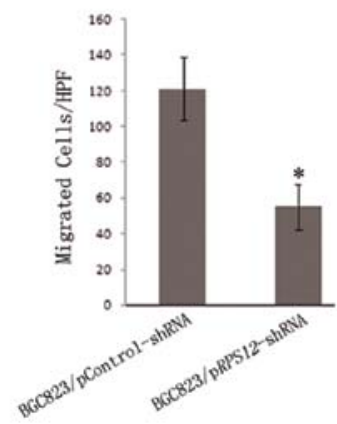

A2

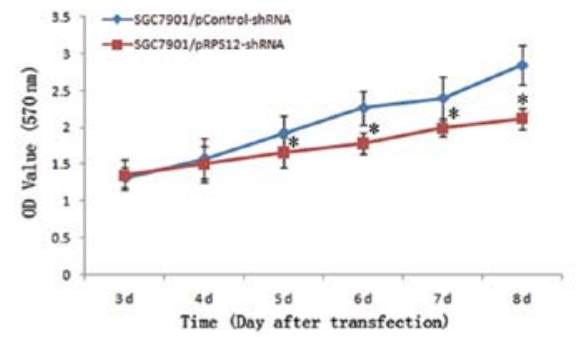

B2

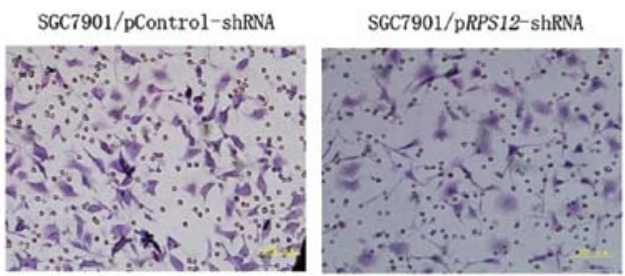

$\mathrm{C} 2$

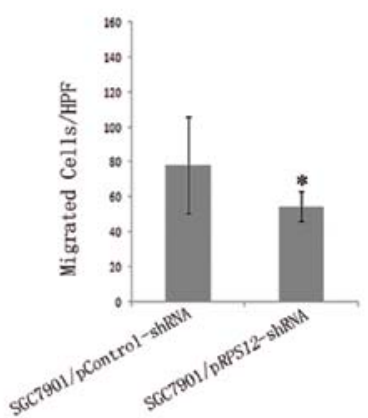

Figure 2. Downregulation of RPS12 expression inhibits proliferation and migration of gastric cancer cell BGC823 and SGC7901. (A) Proliferation of BGC823/SGC7901/pRPS12-shRNA and BGC823/SGC7901/pControl-shRNA cells was analyzed by (A1-A2) MTT assay as described in Materials and methods. On day 3, 4, 5, 6, 7 and 8 after transfection. "p $<0.05$ vs the corresponding pControl-shRNA cells. (B) The representative pictures of transwell assays for BGC823/ SGC7901/pRPS12-shRNA and BGC823/SGC7901/pControl-shRNA cells (B1 and B2, BGC823 and SGC7901, respectively), as described in Materials and methods. (C) The numbers of migrated cells were plotted as mean from triplicate experiments; " $\mathrm{p}<0.05$ vs the corresponding pControl-shRNA cells (C1 and $\mathrm{C} 2$, BGC823 and SGC7901, respectively). Bars indicate SD. HPF, high power field (x200).

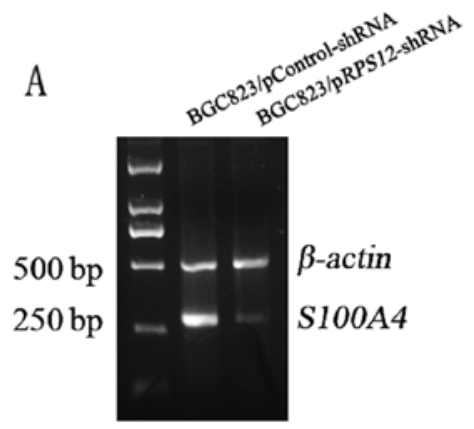

$\mathrm{B}$

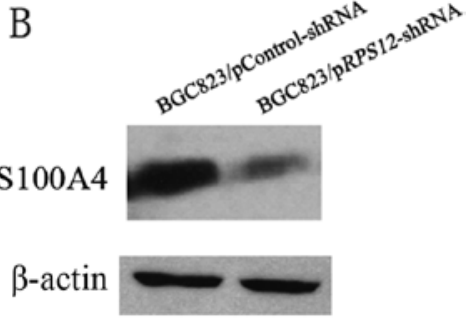

Figure 3. RPS12 inhibition leads to a decrease in S100A4 expression. BGC823/pRPS12-shRNA or BGC823/pControl-shRNA cells were grown under normal culture conditions. RNA and protein were extracted for (A) RT-PCR and (B) western blot analysis on day 5 after pRPS12-shRNA or pControl-shRNA transfection as described in Materials and methods.

whether RPS12 regulates the transcriptional activity of S100A4 promoter, pGL3-S100A4-luc plasmid was transfected into BGC823/pRPS12-shRNA cells or BGC823/pControlshRNA cell, respectively. The results of Dual-Luciferase Reporter Assay showed that luciferase activity of pGL3S100A4-luc plasmid in BGC823/pRPS12-shRNA cells was significantly lower than that in BGC823/pControl-shRNA cells (Fig. 4).
Ectopic expression of S100A4 reverses the effects of RPS12 inhibition on the proliferation, migration of $B G C 823$ cells. Having found that S100A4 is a molecular target of RPS12, we further determined whether $S 100 A 4$ could mediate the cellular effects of RPSI2 by rescue assay. As shown in Fig. 5, transfection of pcDNA3.1-S100A4 into BGC823/pRPS12-shRNA cells led to increased S100A4 expression in the cells at both mRNA and protein level compared with pcDNA3.1-empty vector trans- 


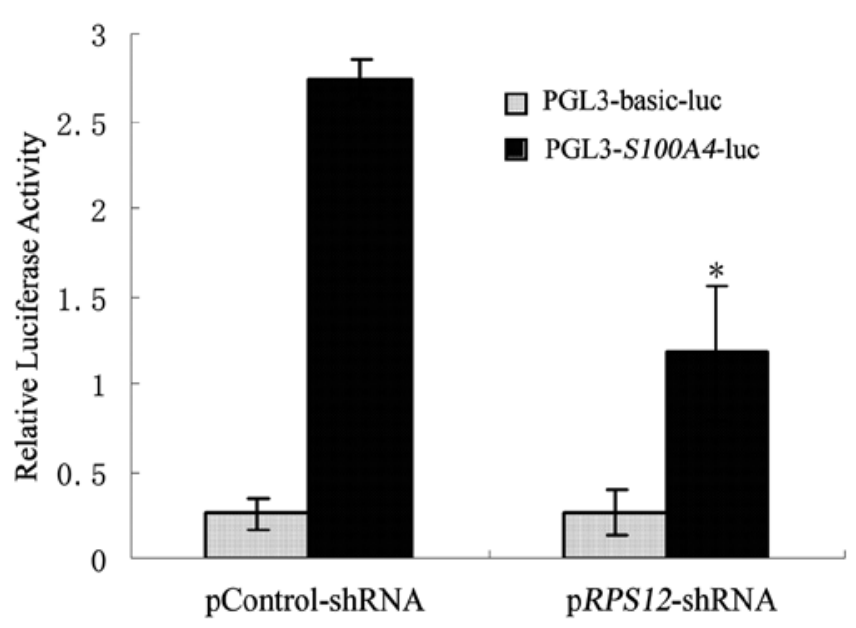

Figure 4. Effect of RPS12 knockdown on the transcriptional activity of S100A4 promoter in BGC823 cells. The relative luciferase activity is represented by the ratio of luciferase activity of firefly (from the various pGL3 vectors) to that of Renilla (from pRL-TK construct, internal control). The data represent the mean \pm SD from three experiments $\left({ }^{*} \mathrm{p}<0.05\right)$.

fection. We then found the following cellular effects. At day 3,4 and 5 after pcDNA3.1-S100A4 transfection, the proliferation of BGC823/pRPS12-shRNA cells was significantly higher than that after pcDNA3.1-empty vector transfection $(\mathrm{p}<0.05)$ (Fig. 6A). The number of migrated cells was significantly increased in BGC823/pRPS12-shRNA cells after
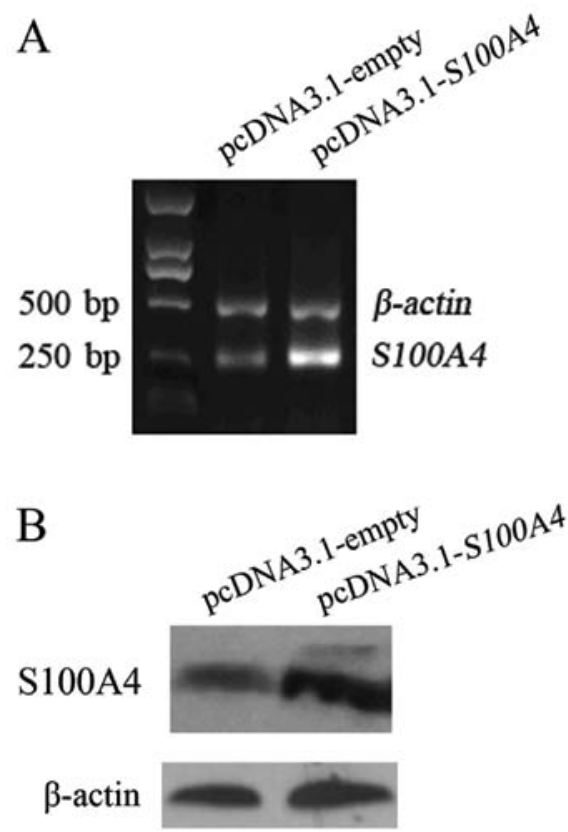

Figure 5. pcDNA3.1-S100A4 transfection enhances S100A4 expression in BGC823/pRPS12-shRNA cells. Expression of S100A4 in BGC823/pRPS12shRNA cells transfected with pcDNA3.1-S100A4 or pcDNA3.1-empty vector was analyzed by (A) RT-PCR and (B) western blot analysis, respectively, as described in Materials and methods.

pcDNA3.1-S100A4 transfection $(97 \pm 11.37)$, as compared to that in BGC823/pRPS12-shRNA cells after pcDNA3.1-empty vector transfection $(49 \pm 8.73)(\mathrm{p}<0.05)$ (Fig. 6B).
A

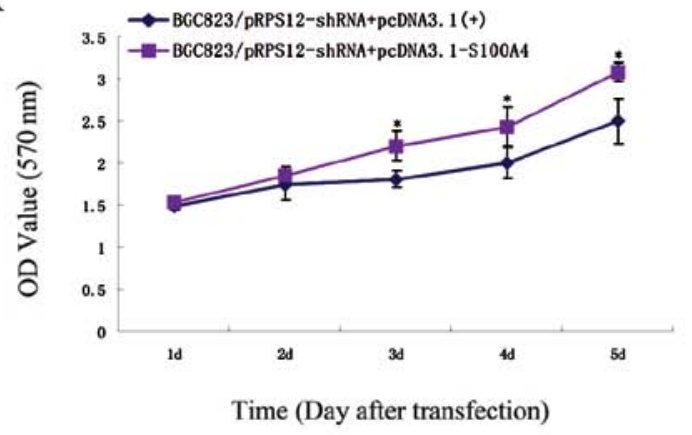

B1

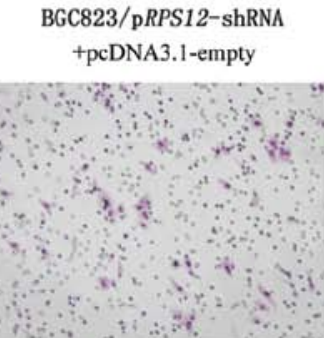
BGC823/pRPS12-shRN + pCDNA3.1-S100A4

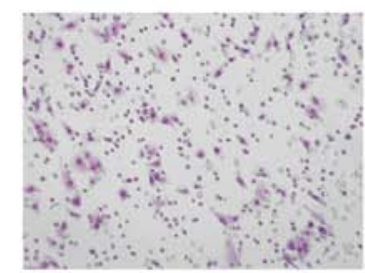

B2

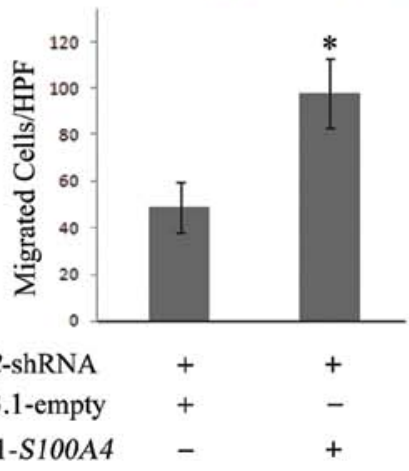

Figure 6. Ectopic expression of S100A4 reverses the effects of RPS12 inhibition on the proliferation, migration of BGC823 cells. (A) Proliferation of BGC823/pRPS12-shRNA cells transfected with pcDNA3.1-S100A4 or pcDNA3.1-empty was analyzed by MTT assay as described in Materials and methods On day 3, 4 and 5 after transfection of pcDNA3.1-S100A4. "p<0.05 vs BGC823/pRPS12-shRNA cells transfected with pcDNA3.1-empty. (B) Migration of BGC823/pRPS12-shRNA cells transfected with pcDNA3.1-S100A4 or pcDNA3.1-empty was analyzed by transwell assays as described in Materials and methods. (B1) Representative pictures of transwell assays. (B2) The number of migrated cells was plotted as mean from triplicate experiments; * $\mathrm{p}<0.05 \mathrm{vs}$ BGC823/pRPS12-shRNA cells transfected with pcDNA3.1-empty; HPF, high power field (x200). 


\section{Discussion}

The ribosome is an organelle essential for protein synthesis in cells, which is composed of ribosomal RNAs and ribosomal proteins (RPs). At present, approximately 80 different ribosomal proteins are found in eukaryotic ribosomes (7). It is very interesting that the reports showing the involvements of RPs in various tumors are emerging (8-14). Kasai et al (15) reported decreased expression of $10 \mathrm{RP}$ genes and increased expression of 2 RP genes in colorectal cancer compared with normal epithelia. Other reports have also shown the overexpression of RP genes in different types of cancers, such as $S 8, L 12, L 23 a$, $L 27$ and $L 30$ mRNAs in human hepatocellular carcinoma (16), RPL13 in gastric cancer (9), and RPL19 in colorectal cancer (10). Additionally, transcriptional loss of RPL14 was observed in lung and oral cancers (17). Reports on the association of abnormal expression of RPs with tumorigenesis are still increasing. RPS12 gene, located in 6q23.2, encoding a ribosomal protein of 132 amino acids as a component of the 40S subunit of ribosome, was isolated as a clone overexpressed in gastric cancer in our previous studies by SSH. Subsequent experiments showed that its overexpression was associated with lymph node metastasis of gastric cancer $(1,2)$. The enhanced expression of RPSI2 gene has also been reported in colorectal cancer and squamous cell carcinoma of the uterine cervix when compared with normal tissues $(11,18)$. O'Donohue et al reported depletion of RPS12 causes delayed processing of $18 \mathrm{~S}$ ribosomal RNA, although a less severe phenotype is seen for RPS12 than for other small subunit r-proteins (19). However, whether or how the overexpression of RPS12 contributes to the development and progression of gastric cancers is not clear.

We carried out the current study to investigate the function of RPS12 in gastric cancer and demonstrated that RPS12 suppression by RNAi led to reduced proliferation and migration in gastric cancer cells BGC823 and SGC7901. The findings indicate that RPS12 may take part in the development, progression and metastasis of gastric cancer or prognosis of the patients by regulating proliferation, migration of gastric cancer cells. Similar to our findings on the cellular effects of RPS12, it has been reported that inhibition of RPL15 expression suppressed the proliferation of gastric cancer cells (20), while RPL44 enhanced colony formation and cell proliferation of hepatocellular carcinoma cells (13). Taken together, the findings above suggest that aberrant expression of certain ribosomal proteins contributes to tumorigenesis and progression of certain types of cancers by regulating biological behavior of the cancer cells.

Based on the important roles of RPSI2 in gastric cancer cells as described above, we try to decipher the mechanisms through which RPS12 performs the functions. Previous studies indicated that ribosomal proteins may exert their roles by regulating the expression of downstream genes. Khanna et al (21) demonstrated that ribosomal protein $S 29$ induced apoptosis in non-small cell lung cancer cells by downregulating $B C L-2, B C L-X L$ and survivin, and also upregulating $P 53$ and $B A X$ expression. It was also reported that $R P L 6$ inhibited cell cycle progression through downregulation of cyclin E (22). Since we found that RPS12 affected many biological characteristics of gastric cancer cells, we investigated whether RPS12 might exert its functions by regulating a multifunctional downstream target gene. S100A4, also known as metastatin-1 (Mts-1), is a member of the S100 family calcium-binding proteins. It is overexpressed in gastric cancer and its expression correlates with poor prognosis of the patients (23-25). It has also been shown that S100A4 inhibition leads to decreased in vitro and in vivo growth of gastric cancer BGC823 cells (4). We previously found that S100A4 suppression leads to decreased migration of BGC823 cells (data not shown). These findings suggest that $S 100 A 4$ can regulate many biological characteristics of gastric cancer cells. We speculated that $S 100 A 4$ may be a downstream effector mediating the role of RPS12. We examined and found that RPS12 inhibition led to decreased expression of $S 100 A 4$ at both mRNA and protein level in BGC823 cells, which indicated that S100A4 might be a downstream target of RPS12. Our further study on luciferase reporter assay showed that RPS12 suppression significantly reduced the activity of $S 100 A 4$ promoter, suggesting the involvement of RPS12 in the transcriptional regulation of S100A4 gene. How RPS12 regulates the transcription of the S100A4 needs to be clarified. Other reports have proved that some ribosomal proteins are gene transcription regulators. An example of RPs as a gene transcription regulator is the mammalian RPL11. It binds to the Myc oncoprotein and inhibits Myc-mediated transcriptional activation of target genes (26). L5, L23 and S7 were shown to bind to MDM2 and inhibit MDM2-mediated p53 ubiquitination and degradation, leading to p53 activation (27-29). Based on these studies, we hypothesize that RPS12 may interact with or affect the stability of certain transcription factors regulating S100A4 expression. In what way RPS12 control S100A4 gene transcription is under investigation by our group.

In order to further investigate whether S100A4 mediates the biological effects of RPSI2 in gastric cancer cells, we carried out rescue assays. The results showed that ectopic expression of S100A4 reversed the reduced migration and proliferation ability, which suggested that as a downstream effector, S100A4 could partly mediate the roles of RPS12 in BGC823 cells.

In summary, the current study investigated the effects of RPS12 on gastric cancer cells and the underlying mechanisms. We demonstrated for the first time that RPSI2 suppression was able to inhibit the proliferation and migration of gastric cancer cells. RPS12 inhibition reduces the activity of S100A4 promoter and leads to the downregualtion of S100A4 expression. Ectopic expression of S100A4 is able to reverse the reduced proliferation, and migration by RPS12 inhibition. Collectively, the evidence suggests that RPS12 may take part in the development and progression of gastric cancer by affecting the proliferation and migration, and the effects may be at least partly mediated by S100A4. RPS12 might be a new potential target for diagnosis and therapy of gastric cancer.

\section{Acknowledgements}

This study was supported by grants from the National Natural Science Foundation of China (no. 30570848) and Liaoning Natural Science Foundation (no. 20102289).

\section{References}

1. Sun XJ, Sun KL, Zheng ZH, Hao DM, Fu WN, Xu HM, Chen JQ and Li XM: Analysis of gene expression profiles for distinct stages of intestinal-type gastric cancer using suppression subtractive hybridization and cDNA microarray. Scand J Gastroenterol 40: 1244-1245, 2005. 
2. Sun XJ, Hao DM, Zheng ZH, Fu H, Xu HM, Wang MX and Sun KL: Screening and analysis of associated genes in the carcinogenesis and progression of gastric cancer. Zhonghua Yi Xue Yi Chuan Xue Za Zhi 22: 31-34, 2005 (In Chinese).

3. Yan Y, Fu H, Hua J, Zhang RX, Chen DQ, Sun KL and Sun XJ: The effects of silencing the RPS12 gene by RNAi on apoptosis of gastric cancer cells and the possible molecular mechanism. Chin J Clin Oncol 35: 1415-1418, 2008 (In Chinese).

4. Hua J, Chen DQ, Fu H, Zhang RX, Shen W, Liu SS, Sun KL and Sun XJ: Short haripin RNA-mediated inhibition of S100A4 promotes apoptosis and suppresses proliferation of BGC823 gastric cancer cells in vitro and in vivo. Cancer Lett 292: 41-47, 2010.

5. Zhang RX, Fu H, Chen DQ, Hua J, Hu YP, Sun KL and Sun XJ: Subcellular distribution of S100A4 and its transcriptional regulation under hypoxic conditions in gastric cancer cell line BGC823. Cancer Sci 101: 1141-1146, 2010.

6. Shen W, Chen DQ, Fu H, Liu SS, Sun KL and Sun XJ: S100A4 protects gastric cancer cells from anoikis through regulation of $\alpha v$ and $\alpha 5$ integrin. Cancer Sci 102: 1014-1018, 2011.

7. Frank J: The ribosome - a macromolecular machine par excellence. Chem Biol 7: R133-R141, 2000.

8. Lai MD and $\mathrm{Xu}$ J: Ribosomal proteins and colorectal cancer. Curr Genomics 8: 43-49, 2007

9. Kobayashi T, Sasaki Y, Oshima Y, Yamamoto H, Mita H, Suzuki H, Toyota M, Tokino T, Itoh F, Imai K and Shinomura Y: Activation of the ribosomal protein L13 gene in human gastrointestinal cancer. Int J Mol Med 18: 161-170, 2006.

10. Huang CJ, Chen CC, Yang SH, Chang CC, Sun HL, Cheng YC, Liu CC, Lin SC and Lin CM: Faecal ribosomal protein L19 is a genetic prognostic factor for survival in colorectal cancer. J Cell Mol Med 12: 1936-1943, 2008

11. Cheng Q, Lau WM, Chew SH, Ho TH, Tay SK and Hui KM: Identification of molecular markers for the early detection of human squamous cell carcinoma of the uterine cervix. Br J Cancer 86: 274-281, 2002.

12. Wang M, Hu Y, Amatangelo MD and Stearns ME: Role of ribosomal protein RPS2 in controlling let-7a expression in human prostate cancer. Mol Cancer Res 9: 36-50, 2011.

13. Kim JH, You KR, Kim IH, Cho BH, Kim CY and Kim DG: Over-expression of the ribosomal protein L36a gene is associated with cellular proliferation in hepatocellular carcinoma Hepatology 39: 129-138, 2004.

14. Sridharan S and Basu A: S6 kinase 2 promotes breast cancer cell survival via AKT. Cancer Res 71: 2590-2599, 2011.

15. Kasai H, Nadano D, Hidaka E, Higuchi K, Kawakubo M, Sato TA and Nakayama J: Differential expression of ribosomal proteins in human normal and neoplastic colorectum. J Histochem Cytochem 51: 567-574, 2003

16. Kondoh N, Shuda M, Tanaka K, Wakatsuki T, Hada A and Yamamoto M: Enhanced expression of S8, L12, L23a, L27 and L30 ribosomal protein mRNAs in human hepatocellular carcinoma. Anticancer Res 21: 2429-2433, 2003.
17. Shriver SP, Shriver MD, Tirpak DL, Bloch LM, Hunt JD, Ferrell RE and Siegfried JM: Trinucleotide repeat length variation in the human ribosomal protein L14 gene (RPL14): localization to $3 \mathrm{p} 21.3$ and loss of heterozygosity in lung and oral cancers. Mutat Res 406: 9-23, 1998.

18. Pogue-Geile K, Geiser JR, Shu M, Miller C, Wool IG, Meisler AI and Pipas JM: Ribosomal protein genes are overexpressed in colorectal cancer: isolation of a cDNA clone encoding the human S3 ribosomal protein. Mol Cell Biol 11: 3842-3849, 1998.

19. O'Donohue MF, Choesmel V, Faubladier M, Fichant G and Gleizes PE: Functional dichotomy of ribosomal proteins during the synthesis of mammalian $40 \mathrm{~S}$ ribosomal subunits. J Cell Biology 190: 853-866, 2010.

20. Wang H, Zhao LN, Li KZ, Ling R, Li XJ and Wang L: Overexpression of ribosomal protein L15 is associated with cell proliferation in gastric cancer. BMC Cancer 6: 91, 2006.

21. Khanna N, Sen S, Sharma H and Singh N: S29 ribosomal protein induces apoptosis in H520 cells and sensitizes them to chemotherapy. Biochem Biophys Res Commun 304: 26-35, 2003.

22. Wu Q, Gou Y, Wang Q, Jin H, Cui L, Zhang Y, He L, Wang J, Nie Y, Shi Y and Fan D: Downregulation of RPL6 by siRNA inhibits proliferation and cell cycle progression of human gastric cancer cell lines. PLoS One 6: e26401, 2011.

23. Forst B, Hansen MT, Klingelhöfer J, Møller HD, Nielsen GH, Grum-Schwensen B, Ambartsumian N, Lukanidin E and Grigorian M: Metastasis-inducing S100A4 and RANTES cooperate in promoting tumor progression in mice. PLoS One 5: e10374, 2010.

24. Kwon YW, Chang IH, Kim KD, Kim YS, Myung SC, Kim MK and Kim TH: Significance of S100A2 and S100A4 expression in the progression of prostate adenocarcinoma. Korean J Urol 51: 456-462, 2010

25. Wang YY, Ye ZY, Zhao ZS, Tao HQ and Chu YQ: High-level expression of S100A4 correlates with lymph node metastasis and poor prognosis in patients with gastric cancer. Ann Surg Oncol 17: 89-97, 2010.

26. Dai MS, Sears R and Lu H: Feedback regulation of c-Myc by ribosomal protein L11. Cell Cycle 6: 2735-2741, 2007.

27. Chen D, Zhang Z, Li M, Wang W, Li Y, Rayburn ER, Hill DL, Wang $\mathrm{H}$ and Zhang R: Ribosomal protein $\mathrm{S} 7$ as a novel modulator of p53-MDM2 interaction: binding to MDM2, stabilization of p53 protein, and activation of p53 function. Oncogene 26: 5029-5037, 2007.

28. Dai MS and Lu H: Inhibition of MDM2-mediated p53 ubiquitination and degradation by ribosomal protein L5. J Biol Chem 279: 44475-44482, 2004.

29. Dai MS, Zeng SX, Jin Y, Sun XX, David L and Lu H: Ribosomal protein L23 activates p53 by inhibiting MDM2 function in response to ribosomal perturbation but not to translation inhibition. Mol Cell Biol 24: 7654-7668, 2004. 